\title{
The Role of Micro fluidic Systems in Biological and Medical Sciences
}

\author{
Ignacio M Helbling* and Julio A Luna \\ Instituto de Desarrollo Tecnológico para la Industria Química (INTEC), Universidad Nacional del Litoral, Argentina
}

Submission: February 27, 2017; Published: March 02, 2017

"Corresponding author: Ignacio M Helbling, Instituto de Desarrollo Tecnológico para la Industria Química (INTEC), Universidad Nacional del Litoral, Centro Científico Tecnológico UNL-CONICET-SANTA FE - Santa Fe, Argentina, Tel-Fax: +54 342 4511597;

Email: ihelbling@santafe-conicet.gov.ar

\begin{abstract}
Micro fluidics is a young discipline. During its beginning, it was mainly an academic field in where researchers study the behavior of fluids at micro scale and how it can be modified with operative and experimental variables. Then, the focus was placed on studying device fabrication process and how to optimize them to lower costs and time, and to enhance system features. After a period of maturation, micro fluidic researchers began to evaluate system usefulness and the possibility of use them in different areas. Micro fluidics became a multidisciplinary field combining concepts of biological and medical sciences and engineering. Diagnostic test, micro particles fabrications, contaminant detection, and medical analyses were first goals. Then, its uses expanded exponentially to other areas opening a world of possibilities. With the advances in miniaturization and material sciences as well as the boom in micro and nanotechnology, manufacturing process became highly precise. New applications in biochemistry, biotechnology, biology and medical sciences were appearing attracting the interest of the industrial sector. Since then, projects are aimed to develop micro fluidic systems with industrial applications. The present contribution describes the characteristics of the three major type of micro fluidic systems, chip-based, capillary-based and paper-based systems. Advantages and limitations of each one are mentioned. In addition, their most important applications in biological and medical sciences are presented.
\end{abstract}

Keywords: Micro Fluidics; Micro Fluidic Systems; Lab-On-A-Chip; Capillary-Based Micro Fluidics; Paper-Based Micro Fluidics

\section{Introduction}

Micro fluidics represent a relatively new field of research and development that combine concepts of different areas like biological and medical sciences and engineering. It was born from the study of fluid behavior at micro scale and with time evolved into a multidisciplinary field. Micro fluidics papers are mainly published in engineering, biology and medicine journals. The basic idea is the manipulation of small volumes of fluids with high precision using integrated micro scale devices. These devices are commonly referred to as lab-on-a-chip (LoC) devices or miniaturized total analysis systems ( $\mu$ TASs). Although micro fluidics tends to be a miniaturization of macro scale, the forces that govern micro scale process are quite different. Capillarity and surface tension are the predominant forces in the micro scale while gravity is almost negligible.

The use of micro fluidics in biotechnology and biomedical applications presents numerous advantages. For example, it allows to reduce the volume of samples required for assays reducing reagents cost. In addition, reactions are faster at micro scale, hence tests using micro fluidics consume less time than traditional trials simplifying medical assay protocols and diagnostic tests. Other great feature is the possibility of scaleup and automate process without major inconveniences, and the superior control of experimental variables during tests and fabrication process. Micro fluidics systems are simple to operate avoiding complex and expensive equipments. Also, working at micro scale, sensitivities are improved enhancing process efficiencies, separation and detection methods, and reducing time. These are only some examples of micro scale benefits compared to traditional techniques.

Advances in miniaturization and material sciences as well as the boom in micro and nanotechnology have led to the improvement of manufacturing process and precision control expanding the possibilities of design and the application fields. In this context, three major micro fluidic systems can be distinguished, chip-based, capillary-based, and paper-based systems. A brief description of each one is presented below as well as their most important applications.

\section{Chip-based systems}

The main type of micro fluidic device is the chip-based system. This comprises a chip having micro channels by which 


\section{Organic and Medicinal Chemistry International Journal}

fluids are driven and in where reactions take place. Channels can have a square or round shape and their dimension range from few micrometers to 800-900 $\mu \mathrm{m}$. Pumps are required to conduct fluids inside channels. They supply solutions in a continuous manner or are used for dosing. Also, micro valves are used to determine the flow direction and/or the mode of movement. In addition, several components can be easily integrated, like filters, check valves, sensors, electrodes, resistances, heating or cooling systems, magnetic fields, detector systems, and many other. The principal advantage of micro fluidics is that miniaturization on chips permits enhancing efficiency and mobility as well as reducing sample and reagent volumes. Examples of chip-based systems are presented in Figure 1. These chips serve as platform for different reactions and assays allowing to integrate the entire biological or chemical laboratory in a single device.

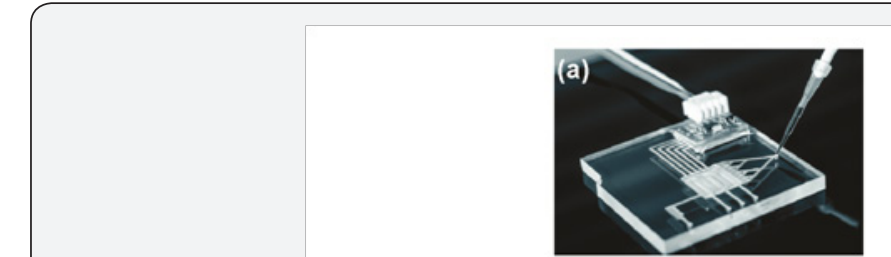

(c)

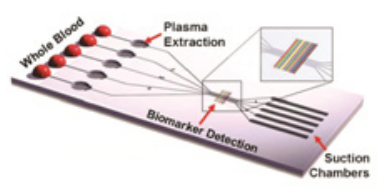

(d)

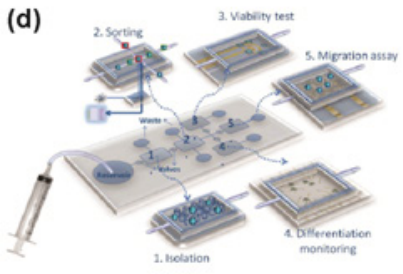

(b)

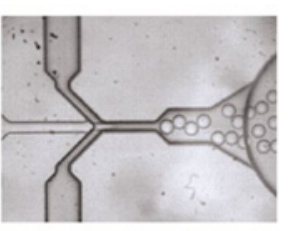

(e)

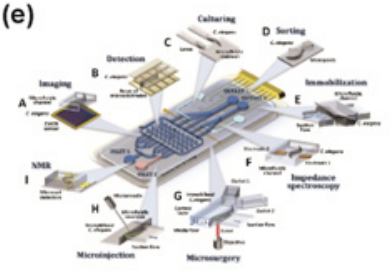

Figure 1: Examples of chip-based systems: (a) Micro fluidic chip [1]. (b) Droplet generation system [2]. (c) Blood analysis system (SIMBAS) [3]. (d) Stem cell analyzer system [4]. (e) Caenorhabditis elegans assaying system [5].

Figure 1 Examples of chip-based systems: (a) Micro fluidic chip [1]. (b) Droplet generation system [2]. (c) Blood analysis system (SIMBAS) [3]. (d) Stem cell analyzer system [4]. (e) Caenorhabditis elegans assaying system [5].

Chip-based systems were first fabricated in silicon [6]. Then, glass was chosen as alternative material [7]. Both type of platform were manufactured using clean-room techniques. As fabrication methods were in general expensive, the popularization of chips was limited. As solution, Duffy et al. [8] developed polydimethylsiloxane (PDMS) chips using soft lithography technique. Using this rapid prototyping technique, fabrication costs were lowered. PDMS is a hydrophobic elastomeric transparent material, which can be easily manipulated with low infrastructure and equipment requirements. Also, its surface can be modified with several treatments to make it hydrophilic [9]. PDMS can stick to glass, plastic and other PDMS structures making it suitable for the preparation of complex systems. In addition, material transparency permits phenomena observation in real time through microscopy. These features made PDMS the most used material for micro fluidics chip fabrication. However, its use has some limitations that can be mentioned. The principal is that it is a vapor permeable material which causes that fluids can evaporate during micro fluidic assays. In addition, it can absorb small molecules and may leach uncross linked oligomers during the curing process generating undesirable effects that could affect the goal application. PDMS limitations promoted the evaluation of new materials as alternative for chip fabrication leading to the use of thermoplastics like polystyrene, polycarbonate, and polymethyl methacrylate [10-12].
Micro fluidic chips have been used in numerous applications, for example as platform for infrared absorption [13], cell culture $[14,15]$, droplet formation [16], digital PCR [17], electrophoresis [18,19] and biosensors [20]. Yu et al. [21] employed chips to separate and sort cells by micro centrifugation. Joshi et al. [22] used them to fabricate liposomes as carrier for pharmaceutical drugs. Good reviews have been reported detailing their use in molecular diagnostics [23], radiobiology [24], and biotechnological applications [25]. In addition, they can be used in enzyme-linked immunosorbent assays (ELISA) and other immunoassays as well as in biochemical analysis and point-ofcare testing [26-28]. Others applications that can be mentioned include micro reactors for chemistry and biological reactions, chemical synthesis, chemical and pharmaceutical analysis, food analysis, genetic analysis, contaminants detection in samples, cellular sample processing, sample processing (concentration, filtration, separation), cell encapsulation, DNA sequencing, and fluidic optics [29].

An interesting organs-on-chips use has been reported recently, in which micro fluidic devices imitate in vivo organ functions allowing researchers to study in depth the effect of drugs, diseases, and active pharmaceutical compounds as well as pharmaceutical formulations [30-34]. This innovative challenge involves the culturing of living cells in continuously per fused chips to mimic physiological conditions of tissues and organs. Systems vary from simpler one-type cell culturing to more complex assemblies for the simultaneous growth of different cell lines. Kidney on, gut, lung, and blood vessels have been simulated with this technology [35-39]. 


\section{Organic and Medicinal Chemistry International Journal}

Another interesting application is in drug delivery field. Chips have been widely employed for manufacturing controlled release systems in the form of micro particles. Manufacturing methodology comprises several steps including:

(i) Formation of micro droplets inside the chip using a polymeric solution containing active principles and a suitable co-solvent to form micro emulsions.

(ii) Micro particle formation by hardening micro droplets caused by one of various phenomena such as solvent extraction, solvent evaporation, UV-mediated polymerization, and phase separation, among others.

(iii) Micro particle separation by usual techniques such as filtration or centrifugation.

(iv) Particle washing.

(v) Particle drying.

By applying this technology, highly mono disperse particles can be obtained with well defined sizes and very low size dispersion. These mono disperse size particles would have a more controlled and defined release rate, thus improving their reproducibility. Batches of particles having different welldefined sizes can be manufactured by varying flow rate ratios between the polymeric solution and the co-solvent, micro channels dimensions and chip configuration. In addition, multilayer particles (coated and sandwich type particles) may be easily synthesized by using the appropriate chip configuration. Good works describing examples of controlled release system fabricated by micro fluidics and their applications can be found in the literature [25,40-46].
The use of lab-on-chip devices presents several advantages such as simplicity, compactness, low manufacturing and operating costs, minimal equipment requirements, precise manipulation and high level of automation, massive parallelization due to compactness, high-throughput, precise control of properties, and great versatility. This promising technology will be probably improved and optimized along time by novel designs and further applications in diverse areas such as biology, medicine, and industry.

\section{Capillary-based systems}

Another type of micro fluidic system is based on the use of capillaries. They are hollow tubes through which the liquid is conducted. Capillaries can be fabricated with different materials, like glass or silica. Fused silica capillaries were first developed for gas chromatography but their key features allowed to expand their use to the fabrication of micro fluidic systems. Systems are formed assembling capillaries with different connectors like simple and tee unions, Y-unions and crosses. This allows to built numerous circuits arranged in different configurations to provide solutions to the challenges posed. Adapters made of inert and resistant materials are used to join capillaries with unions. Example of these is PEEK and stainless steel adapters. In addition, external pumps are required to drive fluids through circuit. The uses of capillaries provide a versatile and durable alternative. They can be constructed with different inner and outer diameters allowing to attend specific requirements. Besides, inner surface can be modified to add new and specific features. Some examples of capillary-based systems are presented in Figure 2.

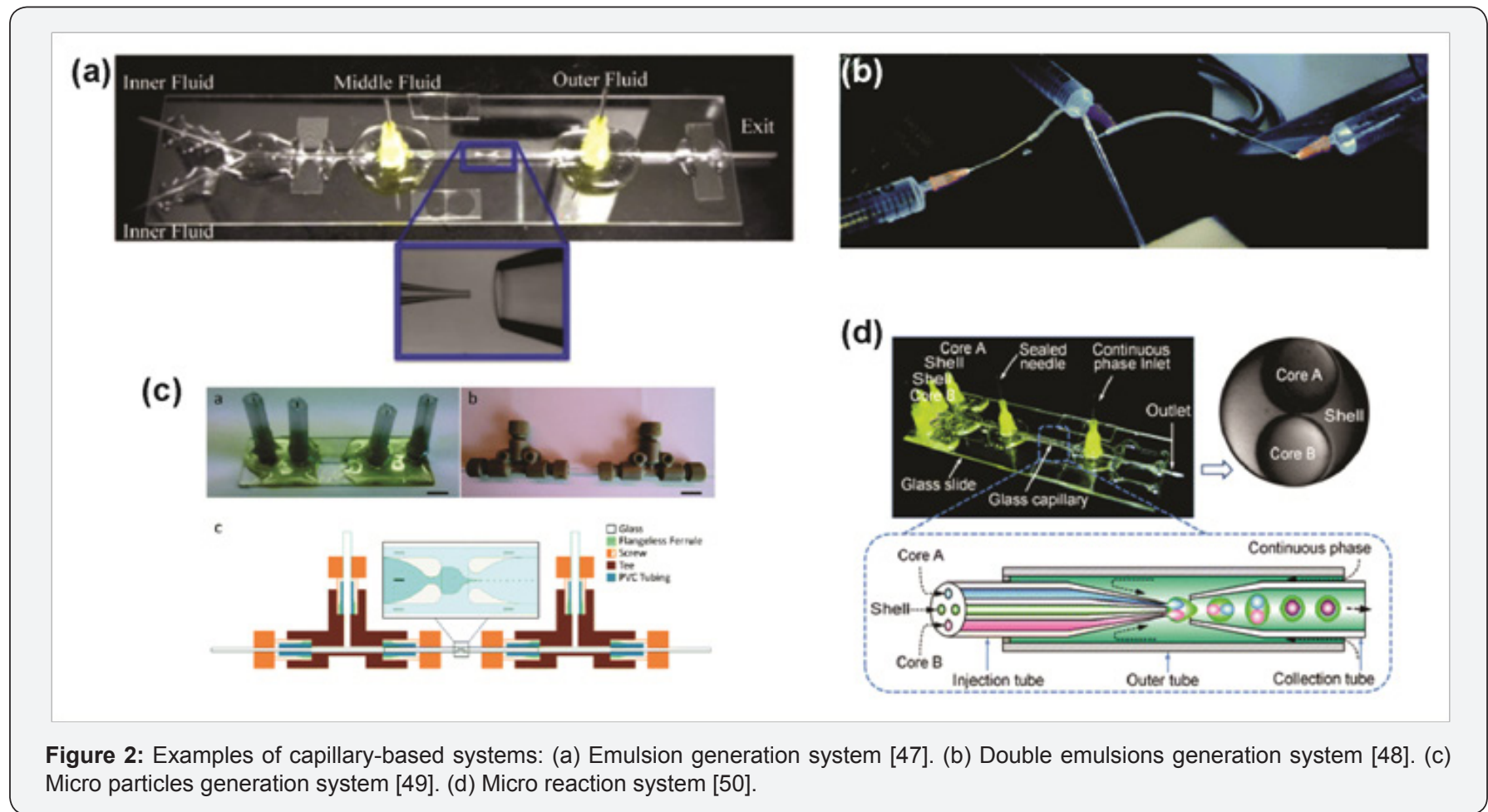




\section{Organic and Medicinal Chemistry International Journal}

Examples of capillary-based systems: (a) Emulsion generation system [47]. (b) Double emulsions generation system [48]. (c) Micro particles generation system [49]. (d) Micro reaction system [50].

Capillary-based systems have been used mainly to generate simple and double emulsions $[47,18,51,52]$. In addition, there are numerous examples of using these systems to prepare micro and nano particles for the controlled release of active pharmaceutical compounds [49,51,53-57]. A good review describing their use as a tool in advanced drug delivery systems fabrication has been reported [58]. In an interesting study, Khan et al. [59] prepared micro particles sensitive to environmental $\mathrm{pH}$, which permits to modulate release rate by $\mathrm{pH}$ changes. Another example is the work of Song et al. [60] which describe the fabrication of narrow size-distribution catalyst beads using capillaries. In all of these applications, droplet and particle dimensions can be modified varying solution flow rates and capillary dimensions, and in some conditions sizes can be predicted from fluid flow profiles. Others uses of these systems include chromatographic applications [61], and to separate substances from water samples [62].

There are several advantages of capillary-based systems. The first that can be noted is their great robustness and durability. Capillaries are made of resistant materials that may be used numerous times and in presence of a large variety of solvents. Also, experimental conditions like temperature, humidity and $\mathrm{pH}$ do not affect their structure and integrity. Other advantage is that these systems present good versatility. Capillaries with different inner and outer diameter and connector with several shapes are commercially available allowing to design numerous combinations of them ranging from simpler systems to complex circuits. It is important to note that all required components are standard and commercially available and the limitation is only on the creativity of researchers. The great distinctive feature is that all systems can be easily disassembled and cleaned between uses without part damage or wear. This represents the most advantage compared with chip-based systems. In addition, they may be easily automated and scale-up by parallelization making easier their adoption by the industry. Future researches must be directed to expand capillaries applications in biological and medical sciences.

\section{Paper-based systems}

Paper-based systems has gained an increasingly interest by the scientific community in recent years. They are composed by paper supports with hydrophilic micro channels through which fluids are conducted. In addition, hydrophobic channels can be constructed using several techniques like printing and photolithographic [63-65]. The operation of these systems is based on the principle of lateral flow assays, pumping fluids passively through patterned channels by capillary forces. The rate of pumping varies with micro channels dimensions, the characteristics of the paper, and the characteristics (temperature and relative humidity) of the environment. Channels can be designed with different patterns to attend application requirements. Initially, they were built in two-dimensional space but in recent years three-dimensional patterns have been popularized. Resulting channels can be left open to the atmosphere or sealed with polymer sheets. Also, surface can be modified by chemical reaction incorporating a wide variety of functional groups to add new features. Examples of paper-based systems are presented in Figure 3.

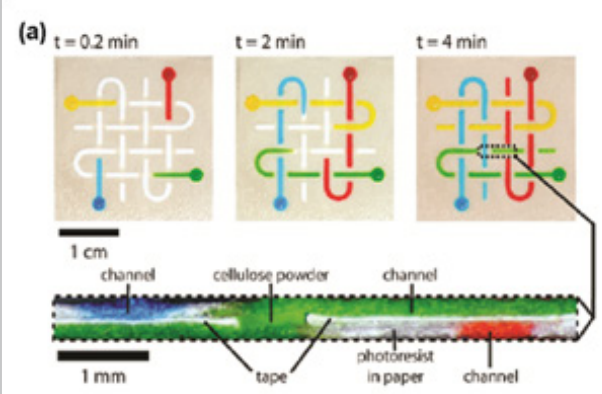

(c)
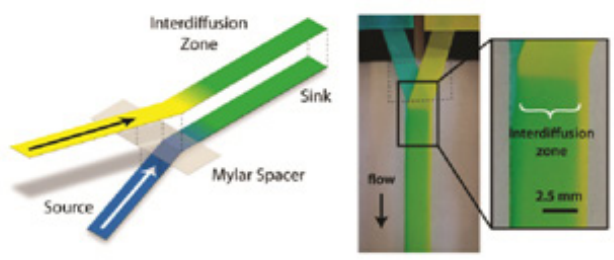

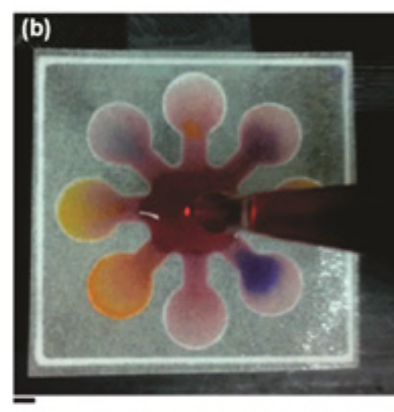

(d)

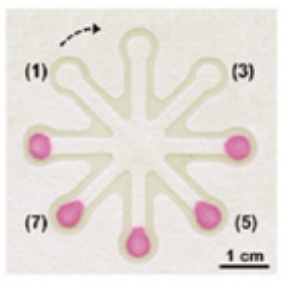

(e)

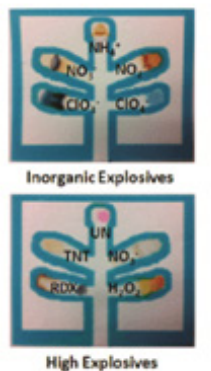

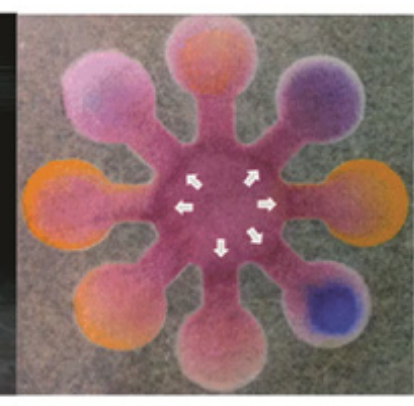

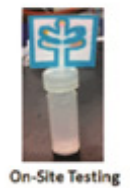

On-Site Testing

Figure 3: Examples of paper-based system: (a) Three-dimensional patterns system [66]. (b) Red wine analyzer system [67]. (c) Mixing system [68]. (d) Nitrite determination system [69]. (e) Explosives detection system [70]. 


\section{Organic and Medicinal Chemistry International Journal}

Examples of paper-based system: (a) Three-dimensional patterns system [66]. (b) Red wine analyzer system [67]. (c) Mixing system [68]. (d) Nitrite determination system [69]. (e) Explosives detection system [70].

Paper-based systems have numerous attractive features. They are low-cost systems, portable, easy to transport and store, easy to use, with good stability, disposable, and made by a renewable resource. Fluids are pumping by capillarity and evaporation avoiding the use of external equipment. Also, disposal are safe with little negative effect on environment. They are compatible with most of the biochemical and medical applications and can be integrated with smart phone applications to make even more portable.

These low-cost systems have been used to improve disease screening in developing countries, in where lack of adequate infrastructure and limited trained medical professionals are common. Also, they were utilized in point-of-care tests and diagnostic assays, in environmental monitoring as well as in food quality testing. In these applications, colorimetric reactions are the most used method for identification and quantification of molecules with biochemical and/or immunological relevance. It provides an easy way to understand for average users like medical personnel or patients. Other methods like electrochemical, fluorescence, and chemiluminescence's detections may also been used. Paper-based systems have been employed for example in the determination of glucose in urine sample [65], nitrite in saliva [69], electrolytes in tear fluid [71], chloride ions [72], bisphenol A [73], human chorionic gonadotropin (HCG) [74], sulfur dioxide [75], and heavy metal [76]. These tests are faster with low cost and good precision, being an excellent alternative to conventional analyses $[77,78]$ In addition, they were used to grade the taste of wines based on the detection of chemical dyes that allowed to distinguish the grape variety and the oxidation status [67]. An interesting application is in cancer detection [79] and cancer cells monitoring [80]. Other uses are to create mixers [68], concentrate samples [81], manipulate and separate substances [82], and indentify explosives [70]. Besides, goods reviews addressing fabrication techniques and applications can be found in the literature $[11,66,83,84]$. Future investigations would be focused on develop increasingly simple and lowcost fabrication techniques and to explore new applications, expanding the use of paper-based systems.

\section{Conclusion}

During its beginning, micro fluidics was mainly an academic field with researchers intended to study phenomena that govern micro scale process. The goal was to understand the forces that act on fluids at this scale and how they can be utilized to modify fluid behavior. Then, it was directed to investigate the best way to fabricate micro devices. Three main type of systems were developed, each one with its advantages and limitations. Different techniques were studied, taking into account material properties, robustness, usability, and time and cost of fabrication. Works addressing that were published mainly in engineering journals. After a period of maturation, scientific community started to evaluate the usefulness of micro fluidic systems and the possibility of use them in different areas. Applications in biochemistry, medical sciences, biology and biotechnology were appearing drawing the interest of the industrial sector. First applications found place in biology research and diagnostic tests. Then, its use exponentially expanded to other areas opening a world of possibilities. Since then, projects were aimed to develop and evaluate micro fluidic systems with industrial applications. Works began to be published in non-engineering journals, like biological, medical and chemical journals. And this trend continues today.

However, micro fluidics is still a young discipline. And it needs to continue growing, expanding its knowledge bases and applications. New investigations should be performed to extend actual limits, enhancing performance, lowering fabrication costs, founding new materials as device platforms as well as simplifying manufacture methods and automation to solve and facilitate the transference of this technology to the industrial sector. With this aim in view, it is essential that micro fluidic researchers partner with biologist, clinicians, biotechnologists, and physics to collaborate together enabling to complement point of views to make more user-friendly devices. In addition, interactions with end-users will be crucial for a successful technological development and adoption. Creating systems as simple as possible involves more creative and efficient solutions but this can enhance user adoption, who can focuses only on interpreting the results. Adoption of new technology that complements or replaces an existing one is often a slow process, but micro fluidics is on track.

\section{Acknowledgment}

Author wish to express its gratitude to Consejo Nacional de Investigaciones Científicas y Técnicas (CONICET) and to Universidad Nacional del Litoral (UNL) of Argentine for the support granted to this contribution.

\section{Reference}

1. Maxwell A (2016) Micro fluidics and Mass Spectrometry-based Proteomics. Thermo Fisher Scientific Inc.

2. Sim SPC, Kang TG, Yobas L, Holtze C, Weitz DA (2010) The shape of a step structure as a design aspect to control droplet generation in microfluidics. Journal of Micromechanics and Microengineering 20(3): 035010.

3. Dimov IK, Basabe Desmonts L, Garcia Cordero JL, Ross BM, Ricco AJ, et al. (2011) Stand-alone self-powered integrated microfluidic blood analysis system (SIMBAS). Lab on a chip 11: 845-850.

4. Primiceri E, Chiriaco MS, Rinaldi R, Maruccio G (2013) Cell chips as new tools for cell biology--results, perspectives and opportunities. Lab Chip 13(19): 3789-3802.

5. Bakhtina NA, MacKinnon N, Korvink JG (2016) Advanced Microfluidic Assays for Caenorhabditis elegans. INTECH open science. 


\section{Organic and Medicinal Chemistry International Journal}

6. Van Lintel HTG, van De Pol FCM, Bouwstra S (1988) A piezoelectric micropump based on micromachining of silicon. Sensors and Actuators 15(2): 153-167.

7. Harrison DJ, Manz A, Fan Z, Luedi H, Widmer HM (1992) Capillary electrophoresis and sample injection systems integrated on a planar glass chip. Analytical Chemistry 64(17): 1926-1932.

8. Duffy DC, McDonald JC, Schueller OJA, Whitesides GM (1998) Rapid Prototyping of Microfluidic Systems in Poly(dimethylsiloxane). Analytical Chemistry 70(23): 4974-4984.

9. Morra M, Occhiello E, Marola R, Garbassi F, Humphrey P, et al. (1990) On the aging of oxygen plasma-treated polydimethylsiloxane surfaces. Journal of Colloid and Interface Science 137(1): 11-24.

10. Vasiliy NG, Yi Cheng H, Odessa NP, Ronald AF, Po Ki Y (2011) Hot embossing of plastic microfluidic devices using poly(dimethylsiloxane) molds. Journal of Micromechanics and Microengineering 21: 017002.

11. Young EWK, Berthier E, Guckenberger DJ, Sackmann E, Lamers C, et al. (2011) Rapid Prototyping of Arrayed Microfluidic Systems in Polystyrene for Cell-Based Assays. Analytical Chemistry 83(4): 14081417.

12. Wang Y, Balowski J, Phillips C, Phillips R, Sims CE, et al. (2011) Benchtop micromolding of polystyrene by soft lithography. Lab Chip 11(18): 3089-3097.

13. Ebrahimifard R, van den Driesche S, Di Salvo M, Vellekoop MJ (2016) Discrimination of Living Biological Cells by Infrared Absorbance Measurements in a Microfluidics Chip. Procedia Engineering 168: 1471-1474.

14. Keil W, Kutscher LM, Shaham S, Siggia ED (2017) Long-Term HighResolution Imaging of Developing C. elegans Larvae with Microfluidics. Developmental Cell 40(2): 202-214.

15. Peñaherrera A, Payés $C$, Sierra-Rodero $M$, Vega $M$, Rosero G, et al (2016) Evaluation of cell culture in microfluidic chips for application in monoclonal antibody production. Microelectronic Engineering 158: 126-129.

16. Mashaghi S, Abbaspourrad A, Weitz DA, van Oijen AM (2016) Droplet microfluidics: A tool for biology, chemistry and nanotechnology. TrAC Trends in Analytical Chemistry 82: 118-125.

17. Fu Y, Zhou H, Jia C, Jing F, Jin Q, Zhao J, Li G (2017) A microfluidic chip based on surfactant-doped polydimethylsiloxane (PDMS) in a sandwich configuration for low-cost and robust digital PCR. Sensors and Actuators B: Chemical 245: 414-422.

18. Zarabadi AS, Pawliszyn J, Hajialamdari M (2017) Development of a multichannel microfluidic system with Schlieren imaging microscopy for online chip-based moving boundary electrophoresis. Journal of Chromatography A 1484: 93-97.

19. Guijt RM, Armstrong JP, Candish E, Lefleur V, Percey WJ, et al. (2011) Microfluidic chips for capillary electrophoresis with integrated electrodes for capacitively coupled conductivity detection based on printed circuit board technology. Sensors and Actuators B: Chemical 159: 307-313.

20. López MA, Moreno-Guzman M, Jurado B, Escarpa A (2016) Chapter 9 Food Microfluidics Biosensors. In Comprehensive Analytical Chemistry 74: 273-312.

21. Yu ZTF, Joseph JG, Liu SX, Cheung MK, Haffey PJ, Fu J (2017) Centrifugal microfluidics for sorting immune cells from whole blood. Sensors and Actuators B: Chemical.

22. Joshi S, Hussain MT, Roces CB, Anderluzzi G, Kastner E, et al. (2016) Microfluidics based manufacture of liposomes simultaneously entrapping hydrophilic and lipophilic drugs. International Journal of Pharmaceutics 514: 160-168.
23. Jayamohan H, Romanov V, Li H, Son J, Samuel R, Nelson J, Gale BK (2017) Chapter 11 - Advances in Microfluidics and Lab-on-a-Chip Technologies A2 - Patrinos, George P. In Molecular Diagnostics (Third Edition). (Academic Press), pp. 197-217.

24. Lacombe J, Phillips SL, Zenhausern F (2016) Microfluidics as a new tool in radiation biology. Cancer Letters 371(2): 292-300.

25. Konwarh R, Gupta P, Mandal BB (2016) Silk-microfluidics for advanced biotechnological applications: A progressive review. Biotechnology Advances 34(5): 845-858.

26. Ahn CH, Kai J, Lee SH, Santiago N, Sehy DW, et al. (2011) A New Game Changer for Immunoassays and IVD: Microfluidics and Polymer Labon-a-Chips. Procedia Engineering 25: 651-656.

27. Wu J, He Z, Chen Q Lin JM (2016) Biochemical analysis on microfluidic chips. TrAC Trends in Analytical Chemistry 80: 213-231.

28. Mukherji S, Mondal D (2017) 5 - Lab-on-chip (LOC) devices for point of care (POC) applications A2 - Narayan, Roger J. In Medical Biosensors for Point of Care (POC) Applications. (Woodhead Publishing), pp. 99131.

29. Sackmann EK, Fulton AL, Beebe DJ (2014) The present and future role of microfluidics in biomedical research. Nature 507: 181-189.

30. Bhatia SN, Ingber DE (2014) Microfluidic organs-on-chips. Nat Biotech 32: 760-772.

31. Walsh CL, Babin BM, Kasinskas RW, Foster JA, McGarry MJ, et al. (2009) A multipurpose microfluidic device designed to mimic microenvironment gradients and develop targeted cancer therapeutics. Lab Chip 9(4): 545-554.

32. Jimenez Torres JA, Beebe DJ, Sung KE (2016) A Microfluidic Method to Mimic Luminal Structures in the Tumor Microenvironment. Methods Mol Biol 1458: 59-69.

33. Shin K, Klosterhoff BS, Han B (2016) Characterization of Cell-TypeSpecific Drug Transport and Resistance of Breast Cancers Using Tumor-Microenvironment-on-Chip. Mol Pharm 13(7): 2214-2223.

34. Zuchowska A, Kwapiszewska K, Chudy M, Dybko A, Brzozka Z (2017) Studies of anticancer drug cytotoxicity based on long-term HepG2 spheroid culture in a microfluidic system. Electrophoresis 16.

35. Kimura H, Yamamoto T, Sakai H, Sakai Y, Fujii T (2008) An integrated microfluidic system for long-term perfusion culture and on-line monitoring of intestinal tissue models. Lab Chip 8(5): 741-746.

36. Huh D, Matthews BD, Mammoto A, Montoya-Zavala M, Hsin HY, et al. (2010) Reconstituting organ-level lung functions on a chip. Science 328: 1662-1668.

37. Tsai M, Kita A, Leach J, Rounsevell R, Huang JN, et al. (2012) In vitro modeling of the microvascular occlusion and thrombosis that occur in hematologic diseases using microfluidic technology. J Clin Invest 122 : 408-418.

38. Bischel LL, Young EW, Mader BR, Beebe DJ (2013) Tubeless microfluidic angiogenesis assay with three-dimensional endotheliallined microvessels. Biomaterials 34(5): 1471-1477.

39. Jang KJ, Suh KY (2010) A multi-layer microfluidic device for efficient culture and analysis of renal tubular cells. Lab Chip 10: 36-42.

40. Nguyen NT, Shaegh SAM, Kashaninejad N, Phan DT (2013) Design, fabrication and characterization of drug delivery systems based on lab-on-a-chip technology. Advanced Drug Delivery Reviews 65(11-12): 1403-1419.

41. Luo G, Du L, Wang Y, Lu Y, Xu J (2011) Controllable preparation of particles with microfluidics. Particuology 9(6): 545-558.

42. Zhao CX (2013) Multiphase flow microfluidics for the production of 


\section{Organic and Medicinal Chemistry International Journal}

single or multiple emulsions for drug delivery. Advanced Drug Delivery Reviews 65(11-12): 1420-1446.

43. Hong S, Hsu HJ, Kaunas R, Kameoka J (2012) Collagen microsphere production on a chip. Lab on a Chip 12(11): 3277-3280.

44. Lan W, Li S, Xu J, Luo G (2011) Controllable preparation of nanoparticlecoated chitosan microspheres in a co-axial microfluidic device. Lab on a Chip 11(4): 652-657.

45. Chung BG, Lee KH, Khademhossein, A, Lee SH (2012) Microfluidic fabrication of microengineered hydrogels and their application in tissue engineering. Lab on a Chip 12: 45-59.

46. Krutkramelis K, Xia B, Oakey J (2016) Monodisperse polyethylene glycol diacrylate hydrogel microsphere formation by oxygen-controlled photopolymerization in a microfluidic device. Lab on a Chip 16: 14571465.

47. Utada AS, Lorenceau E, Link DR, Kaplan PD, Stone HA, et al. (2005) Monodisperse double emulsions generated from a microcapillary device. Science 308: 537-541.

48. Nurumbetov G, Ballard N, Bon SAF (2012) A simple microfluidic device for fabrication of double emulsion droplets and polymer microcapsules. Polymer Chemistry 3: 1043-1047.

49. Benson BR, Stone HA, Prud'homme RK (2013) An "off-the-shelf" capillary microfluidic device that enables tuning of the droplet breakup regime at constant flow rates. Lab on a chip 13(23): 4507-4511.

50. Chen H, Zhao Y, Li J, Guo M, Wan J, et al. (2011) Reactions in double emulsions by flow-controlled coalescence of encapsulated drops. Lab on a chip 11(14): 2312-2315.

51. Herranz Blanco B, Ginestar E, Zhang H, Hirvonen J, Santos HA (2017) Microfluidics platform for glass capillaries and its application in droplet and nanoparticle fabrication. International Journal of Pharmaceutics 516(1-2): 100-105.

52. Nabavi SA, Vladisavljević GT, Gu S, Ekanem EE (2015) Double emulsion production in glass capillary microfluidic device: Parametric investigation of droplet generation behaviour. Chemical Engineering Science 130(7): 183-196.

53. Vladisavljević GT, Shahmohamadi H, Das DB, Ekanem EE, Tauanov Z, et al. (2014) Glass capillary microfluidics for production of monodispersed poly (dl-lactic acid) and polycaprolactone microparticles: Experiments and numerical simulations. Journal of Colloid and Interface Science 418: $163-170$

54. Othman R, Vladisavljević GT, Nagy ZK (2015) Preparation of biodegradable polymeric nanoparticles for pharmaceutical applications using glass capillary microfluidics. Chemical Engineering Science 137: 119-130.

55. Othman R, Vladisavljević GT, Hemaka Bandulasena HC, Nagy ZK (2015) Production of polymeric nanoparticles by micromixing in a co-flow microfluidic glass capillary device. Chemical Engineering Journal 280: 316-329.

56. Kim MK, Kim MA, Jenjob R, Lee DH, Yang SG (2016) Capillary microfluidics-derived doxorubicin-containing human serum albumin microbeads for transarterial chemoembolization of hepatic cancer Mater Sci Eng C Mater Biol Appl 62: 391-397.

57. Othman R, Vladisavljević GT, Thomas NL, Nagy ZK (2016) Fabrication of composite poly(d,l-lactide)/montmorillonite nanoparticles for controlled delivery of acetaminophen by solvent-displacement method using glass capillary microfluidics. Colloids and Surfaces B: Biointerfaces 141: 187-195.

58. Fontana F, Ferreira MPA, Correia A, Hirvonen J, Santos HA (2016) Microfluidics as a cutting-edge technique for drug delivery applications. Journal of Drug Delivery Science and Technology 34: 76-87.
59. Khan IU, Stolch L, Serra CA, Anton N, Akasov R (2015) Microfluidic conceived $\mathrm{pH}$ sensitive core-shell particles for dual drug delivery. International Journal of Pharmaceutics 478: 78-87.

60. Song ZF, Wei J, Li X, Zhou WY, Chang ZQ et al. (2014) Synthesis of sizecontrolled Pt/C/PTFE hydrophobic catalyst pellets in a capillary-based microfluidic system. International Journal of Hydrogen Energy 39(30): 16944-16952.

61. Gilar M, McDonald TS, Gritti F (2016) Experimental evaluation of chromatographic performance of capillary and microfluidic columns with linear or curved channels. Journal of Chromatography A 1470: 76-83.

62. Tak V, Kabra A, Pardasani D, Goud DR, Jain R, Dubey DK (2015) A glass capillary based microfluidic electromembrane extraction of basic degradation products of nitrogen mustard and VX from water. Journal of Chromatography A 1426: 16-23.

63. Carrilho E, Martinez AW, Whitesides GM (2009) Understanding Wax Printing: A Simple Micropatterning Process for Paper-Based Microfluidics. Analytical Chemistry 81(16): 7091-7095.

64. Martinez AW, Phillips ST, Wiley BJ, Gupta M, Whitesides GM (2008) FLASH: A rapid method for prototyping paper-based microfluidic devices. Lab on a chip 8(12): 2146-2150.

65. Abe K, Suzuki K, Citterio D (2008) Inkjet-Printed Microfluidic Multianalyte Chemical Sensing Paper. Analytical Chemistry 80(18): 6928-6934.

66. Li X, Ballerini DR, Shen W (2012) A perspective on paper-based microfluidics: Current status and future trends. Biomicrofluidics 6 .

67. San Park T, Baynes C, Cho SI, Yoon JY (2014) Paper microfluidics for red wine tasting. RSC Advances 4: 24356-24362.

68. Osborn JL, Lutz B, Fu E, Kauffman P, Stevens DY, et al. (2010) Microfluidics without pumps: reinventing the T-sensor and $\mathrm{H}$-filter in paper networks. Lab Chip 10(20): 2659-2665.

69. Cardoso TMG, Garcia PT, Coltro WKT (2015) Colorimetric determination of nitrite in clinical, food and environmental samples using microfluidic devices stamped in paper platforms. Analytical Methods 7: 7311-7317.

70. Peters KL, Corbin I, Kaufman LM, Zreibe K, Blanes L, et al. (2015) Simultaneous colorimetric detection of improvised explosive compounds using microfluidic paper-based analytical devices ([small mu ]PADs). Analytical Methods 7: 63-70.

71. Yetisen AK, Jiang N, Tamayol A, Ruiz Esparza GU, Zhang YS, et al. (2017) Paper-based microfluidic system for tear electrolyte analysis. Lab on a chip.

72. Ding J, He N, Lisak G, Qin W, Bobacka J (2017) Paper-based microfluidic sampling and separation of analytes for potentiometric ion sensing. Sensors and Actuators B: Chemical 243: 346-352.

73. Kong Q, Wang Y, Zhang L, Ge S, Yu J (2017) A novel microfluidic paperbased colorimetric sensor based on molecularly imprinted polymer membranes for highly selective and sensitive detection of bisphenol A. Sensors and Actuators B: Chemical 243: 130-136.

74. Cao L, Fang C, Zeng R, Zhao X, Jiang Y, et al. (2017) Paper-based microfluidic devices for electrochemical immunofiltration analysis of human chorionic gonadotropin. Biosensors and Bioelectronics 92: 8794.

75. Liu CC, Wang YN, Fu LM, Yang DY (2017) Rapid integrated microfluidic paper-based system for sulfur dioxide detection. Chemical Engineering Journal 316: 790-796.

76. Lin Y, Gritsenko D, Feng S, Teh YC, Lu X, et al. (2016) Detection of heavy metal by paper-based microfluidics. Biosensors and Bioelectronics 83: 256-266. 


\section{Organic and Medicinal Chemistry International Journal}

77. von Lode P (2005) Point-of-care immunotesting: Approaching the analytical performance of central laboratory methods. Clinical Biochemistry 38(7): 591-606.

78. Martinez AW, Phillips ST, Whitesides GM, Carrilho E (2010) Diagnostics for the developing world: microfluidic paper-based analytical devices Anal Chem 82: 3-10.

79. Wang Y, Xu H, Luo J, Liu J, Wang L, et al. (2016) A novel labelfree microfluidic paper-based immunosensor for highly sensitive electrochemical detection of carcinoembryonic antigen. Biosensors and Bioelectronics 83: 319-326.

80. Liang L, Su M, Li L, Lan F, Yang G, et al. (2016) Aptamer-based fluorescent and visual biosensor for multiplexed monitoring of cancer cells in microfluidic paper-based analytical devices. Sensors and Actuators B: Chemical 229: 347-354.
81. Phan DT, Shaegh SAM, Yang C, Nguyen NT (2016) Sample concentration in a microfluidic paper-based analytical device using ion concentration polarization. Sensors and Actuators B: Chemical 222: 735-740.

82. Zhong ZW, Wu RG, Wang ZP, Tan HL (2015) An investigation of paper based microfluidic devices for size based separation and extraction applications. Journal of Chromatography B 1000: 41-48.

83. Xia Y, Si J, Li Z (2016) Fabrication techniques for microfluidic paperbased analytical devices and their applications for biological testing: A review. Biosensors and Bioelectronics 77: 774-789.

84.Ahmed S, Bui MPN, Abbas A (2016) Paper-based chemical and biological sensors: Engineering aspects. Biosensors and Bioelectronics 77: 249-263.

Your next submission with Juniper Publishers will reach you the below assets

- Quality Editorial service

- Swift Peer Review

- Reprints availability

- E-prints Service

- Manuscript Podcast for convenient understanding

- Global attainment for your research

- Manuscript accessibility in different formats ( Pdf, E-pub, Full Text, Audio)

- Unceasing customer service

Track the below URL for one-step submission https://juniperpublishers.com/online-submission.php 